\title{
EDITORIAL
}

\section{Evaluating the Journal's Quality: Methods and Tools}

Increasing access to information and media made available large number of scientific publications. Nevertheless, the worth of findings needs to be cautiously evaluated before its application. Research findings published in different journals do not offer high quality with equal weight on evidences. Widespread availability of publications put pressure on readers to screen the journals and articles before these are utilized. Therefore, evaluating the quality of academic journals is becoming increasing important in research performance evaluation. In fact "The number does not matter, the quality does" concept is indispensible in scientific world. Understanding the methods and criteria for assessing journal quality is critical in determining the overall value of publications.

Evaluation methods such as peer review; impact factors and reputation of the journal are considered to be the hallmarks of journals. In addition to the traditional measures, journal ranking and article quality assessment are new techniques of evaluation of journal quality. Calculation of Eigenfactor score, SCImago Journal Rank (SJR), Article-Level Metrics and data citation index and; alternative matrices (altmetrics) like blogs, social media and some online systems are also in practice. Most common tools to measure journal impact are Journal Citation Report (JCR) and SCImago Journal Rank (SJR). Important evaluation methods are introduced here in this paper.

1. Peer review: It is the evaluation of work by one or more people with similar competences. It is commonly accepted as an essential part of scientific publication. It is often used to determine an academic paper's suitability for publication and to validate academic work. The review may be single blind, double blind and open review.

2. Impact factors: The impact factor (IF) is a measure of the frequency with which the average article in a journal has been cited in a particular year. It is used to measure the importance of a journal by calculating the times its articles are cited. Calculation of IF is based on a twoyear period and involves dividing the number of times articles were cited by the number of articles that are citable.

Journal Citation Reports provides ranking for journals in the areas of science, technology, and social sciences. Citation and article counts, Impact factor, Immediacy index, Cited half-life, citing half-life, source data listing, citing journal listing, cited journal listing, subject categories, publisher information are collected and counted in JCR. JCR includes two editions: the Science Edition and the Social Sciences Edition.

Eigenfactor score and Article Influence score: Like impact factor, the Eigenfactor score and Article Influence score use citation data to assess and track the influence of a journal in relation to other journals. The Eigenfactor score calculation is based on the number of times articles from the journal published in the past five years have been cited in the JCR year. The Article Influence determines the average influence of a journal's articles over the first five years after publication. It is calculated by dividing a journal's Eigenfactor Score by the number of articles in the journal. Thus, Eigenfactor scores are intended to give a measure of how likely a journal is to be used, and how frequently an average researcher would access content from that journal.

3. SCImago Journal Rank (SJR): SCImago Journal Rank is a measure of scientific influence of scholarly journals that accounts for both the number of citations received by a journal and the importance or prestige of the journals where such citations come from. It is the measure of scientific influence of scholarly journals contained in the Scopus database from 1996. 
Difference between Impact factor and SCImago Journal Rank

The main difference between IF and SJR that the IF gives equal weight to all citations, making no distinction between citations published in some obscure journals and citations published in Nature or Lancet. To address this issue, SJR uses mathematical approach behind the Google's Page Rank algorithm and adapts it to journal metrics. The Page Rank model type weights citations from journals according to how highly cited the journal itself is.

Furthermore, impact factors are derived from citations in a single year to articles from the two preceding years whereas SJR looks at citations made in a three year period. This makes the SJR a more stable indicator of trends than impact factors, which often fluctuate substantially from year to year.

\section{Other metrics}

1. Google Scholar Metrics: It provides an easy way for authors to quickly gauge the visibility and influence of recent articles in scholarly publications. It summarizes recent citations to many publications, to help authors as they consider where to publish their new research.

\section{Article-Level Metrics (ALM): Many} other methods of measuring the impact of published articles involve metrics computed at the article or author level rather than the journal level. It demonstrates the impact of an individual article. Most important reason for article level assessment is to promote speed of feedback and superior relationship mapping and influence tracking of the article.

3. PLoS Article Level Metrics: Since March 2009, PLOS introduced article level matrices for all articles including downloads, citations and altmetrics. These ALMs present the broad range of snapshots and cumulative information including HTML page views, PDS downloads, XML downloads Pub Med central usage, citations, Google scholars, Scopus, social media like face book, twitter and blogs.

4. Scopus: It is a large abstract and citation data base. The inclusion of ALMs expands the range of information that indexes traditionally. Data sources include social media sites such as twitter, face book, Pinterest and Google+ and scientific blogs; reference manager and bookmarking sites (e.g. mendly, citeULike).

\section{Alternative matrices (altmetrics):}

Altmetrics are non-traditional bibliometrics to compliment traditional citation impacts. The term "altmetrics" was proposed in 2010 , as a generalization of article level matrics. Altmetrics do not cover citation counts but calculate scholar impact based on diverse online research output, such as social media, online news media, and online reference managers. It demonstrates both the impact and the detailed composition of the impact.

6. Impact Story: It is an open-source altmetric tool. It draws from a variety of social and data sources, including Facebook, Twitter, CiteULike, Delicious, PubMed, Scopus, Cross Ref, scienceseeker, Mendeley, Wikipedia, slideshare, Dryad and figshare. It normalize metrics based on sample of article published in the same year. Impact Story offers a wide range of free widget to embed matrics on any web page.

7. Plum Analytics: It is Seattlebased technology startup. It aims to track metrics for nearly two dozen outputs including journal articles, book chapters, data sets, presentations and course code. It provides customer reports intended to quantify productivity, support grant proposal and address impact related questions.

8. Data Citation Index (DCI): DCI was introduced by Thomson Reuters in 2012 as a Single point of access to quality research data from repositories across disciplines and around the world. Data Citation Index is a searchable collection of data sets and data studies from a select list of repositories. DCI is intended to facilitate the discovery of data, link data to the 
literature, and encourage citation of data. In this index, descriptive records are created for data objects and linked to literature articles in the Web of Science.

\section{REFERENCES}

1. John Minger, Yang Liying. Evaluating journal quality: A review of journal citation indicators and ranking in business and management. European Journal of Operational Research;257 (1): Pages 323-337

2. Boston College Libraries. Assessing Journal Quality: Other metrics. Available from: https:// libguides.bc.edu/journalqual. Cited on 22 February 2019.

3. University Library (UIC). Measuring Your Impact: Impact Factor, Citation Analysis, and other Metrics: Journal Impact Factor (IF). Available from: https://researchguides.uic.edu/ if/impact. Cited on 22 February 2019.
4. Greg Tananbaum. Article-Level Metrics. A SPARC Primer. Available from https:// sparcopen.org/our-work/article-level-metrics/. Cited on 22 February 2019.

5. SCImago Journal \& Country Rank. "Description of Scimago Journal Rank Indicator"SCImago Journal \& Country Rank. Retrieved on 23 February 2019.

6. PLOS. A Comprehensive Assessment of Impact with Article-Level Metrics (ALMs). Available from: https://www.plos.org/articlelevel-metrics. Retrieved on 23 February 2019.

7. Laura LP. Data Citation Index. Journal of the Medical Library Association;104(1):88-90.

\section{Correspondence:}

Dr. Damaru Prasad Paneru,

Editor-in-Chief, Journal of Health and Allied Sciences

E-mail: damaru.paneru@gmail.com 